\title{
Continuous Mid-Infrared Star Formation Rate Indicators
}

\author{
A. J. Battisti ${ }^{1}$, D. Calzetti ${ }^{1}$, B. D. Johnson ${ }^{2}$ and D. Elbaz ${ }^{3}$ \\ ${ }^{1}$ Department of Astronomy, University of Massachusetts, Amherst, MA 01003, USA \\ email: abattist@astro.umass.edu \\ ${ }^{2}$ Institut d'Astrophysique de Paris, UMR 7095, 75014, Paris, France \\ ${ }^{3}$ Laboratoire AIM-Paris-Saclay, CEA/DSM/Irfu, CNRS, Université Paris Diderot, \\ Saclay, pt courrier 131, 91191 Gif-sur-Yvette, France
}

\begin{abstract}
We present continuous, monochromatic star formation rate (SFR) indicators over the mid-infrared wavelength range of $6-70 \mu \mathrm{m}$. We use a sample of 58 star forming galaxies (SFGs) in the Spitzer-SDSS-GALEX Spectroscopic Survey (SSGSS) at $z<0.2$, for which there is a rich suite of multi-wavelength photometry and spectroscopy from the ultraviolet through to the infrared. The data from the Spitzer infrared spectrograph (IRS) of these galaxies, which spans $5-40 \mu \mathrm{m}$, is anchored to their photometric counterparts. The spectral region between $40-70 \mu \mathrm{m}$ is interpolated using dust model fits to the IRS spectrum anchored by Spitzer 70 and $160 \mu \mathrm{m}$ photometry. Since there are no sharp spectral features in this region, we expect these interpolations to be robust. This spectral range is calibrated as a SFR diagnostic using several reference SFR indicators to mitigate potential bias. Our band-specific continuous SFR indicators are found to be consistent with monochromatic calibrations in the local universe, as derived from Spitzer, WISE, and Herschel photometry. Additionally, in the era of the James Webb Space Telescope this will become a flexible tool, applicable to any SFG up to $z \sim 3$.
\end{abstract}

Keywords. galaxies: star formation - infrared: galaxies - stars: formation

\section{Introduction}

Star formation is a fundamental parameter of galaxies that gives key constraints on how galaxies are able to form and evolve (Somerville et al. 2012). For this reason, great efforts have been made to calibrate a wide range of the electromagnetic spectrum that can be linked to processes involved with recent star formation (see review by Kennicutt \& Evans 2012). In particular, infrared (IR) wavelength calibrations are proving to be critical to understanding galaxies in the early Universe. However, difficulties arise in utilizing local calibrations because the regions of rest-frame wavelengths probed by a given band will vary with redshift. Therefore, in order to fully utilize current and future deep IR imaging surveys, continuous single-band SFR indicators will be imperative.

\section{Results}

After anchoring the spectroscopy and dust models of Draine \& Li (2007) to the global IR photometric values, we normalize each spectrum by its reference SFR. This reference value is taken to be the average of the calibrations of Kennicutt et al. (2009), Rieke et al. (2009), Calzetti et al. (2010), and Hao et al. (2011), which incorporate the full suite of data available for this sample. The SFR-normalized IRS spectra are averaged together to create a composite spectrum for the group (see Figure 1). The SFR-normalized IRS spectra are also convolved with the filter response of specific bands as functions of redshift (see Figure 1). Two of the wavelength-continuous calibrations are shown in Figure 2, 

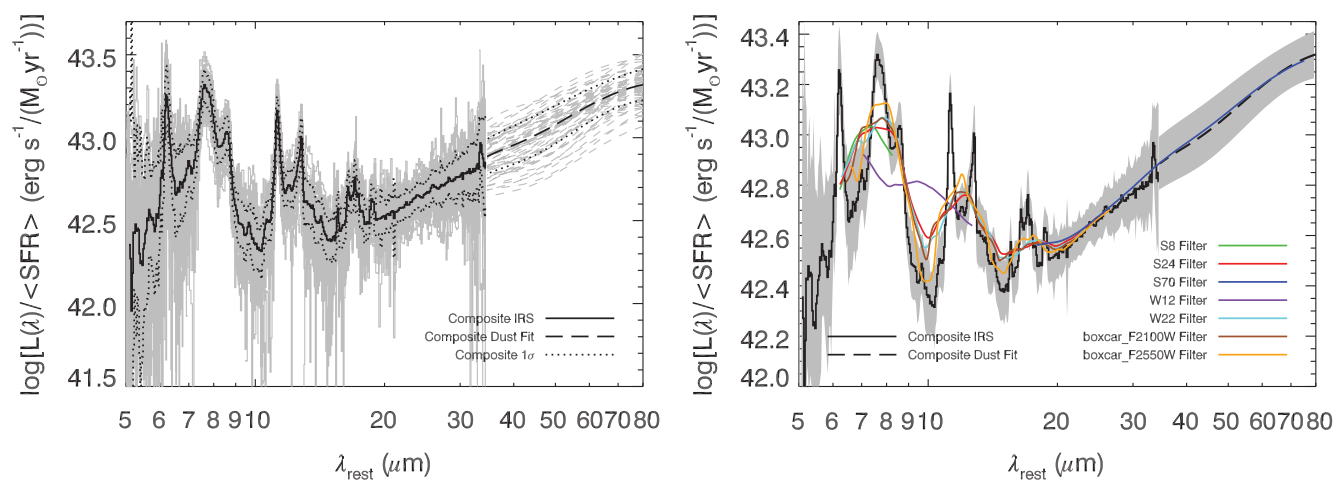

Figure 1. Left: Normalized IRS luminosity, $L(\lambda) /\langle S F R\rangle$, for our SFG galaxies (gray solid lines). The composite spectrum is the thick black line and the standard deviation from of this average is the black dotted line. The dust continuum fit for each galaxy (gray dashed lines) along with the average (black dashed line) and $1 \sigma$ standard deviation is also shown. Right: The solid colored lines show the composite after each spectrum is convolved with the Spitzer, WISE, and JWST-MIRI filters as functions of redshift.
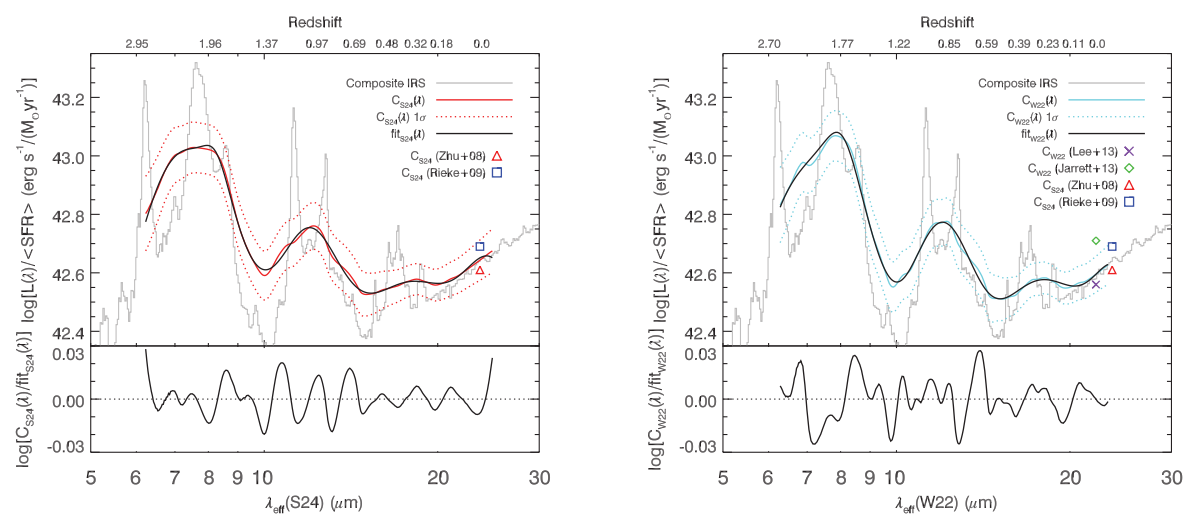

Figure 2. Example of the conversion factor for a Spitzer and WISE band using our SFG galaxies (solid colored lines), along with their uncertainty (dotted colored lines). The solid black line is the fit to this conversion, $f_{i t}(\lambda)$. The local conversion factors from the literature are also shown for comparison (colored symbols).

and they demonstrate that our calibrations are typically accurate to within $30 \%$. It is important to emphasize that the accuracy of such an application is dependent on the shape of the SED of SFGs as a function of redshift. The extent to which this condition holds is being examined in detail.

\section{References}

Calzetti, D., Wu, S.-Y., Hong, S., et al. 2010, ApJ, 714, 1256

Draine, B. T. \& Li, A. 2007, ApJ, 657, 810

Hao, C.-N., Kennicutt, R. C., Johnson, B. D., Calzetti, D., et al. 2011, ApJ, 741, 124

Kennicutt, R. C., Jr. \& Evans, N. J., II 2012, ARA\&A, 50, 531

Kennicutt, R. C., Hao, C.-N., Calzetti, D., et al. 2009, ApJ, 703, 1672

Rieke, G. H., Alonso-Herrero, A., Weiner, B. J., et al. 2009, ApJ, 692, 556

Somerville, R. S., Gilmore, R. C., Primack, J. R., \& Domínguez, A. 2012, MNRAS 423, 1992 